\title{
LIGHT SCALAR NEUTRINOS AND MUON DECAY
}

W. Buchmiiller and F. Scheck ${ }^{*}$ )

CERN - Geneva

\section{A B S T R A C T}

We investigate the constraints which muon decay experiments impose on the existence of light scalar neutrinos. If the s-neutrino masses are small compared to the muon mass, the charged wino has to be substantially heavier than the charged W-vector boson. From the existing measurements of the decay parameters $\rho$ and $\delta$ we find $M_{W} / M_{W}>4$. This bound can be improved by almost a factor of 2 by current experiments.

*) Permanent address: Institut für Physik, Johannes Gutenberg-Universität Mainz, 6500 Mainz, WestGermany.

Ref.TH.3933-CERN

June 1984 
1. Supersymmetric extensions /1/ of the standard model of electroweak interactions predict the existence of scalar superpartners of the known quarks and leptons. In most models scalar quarks and leptons are expected to be much heavier than their fermionic superpartners; they are essentially degenerate in mass with the gravitino. Yet it is also conceivable that the scalar mass spectrum is much more complicated. In particular, scalar neutrinos, like ordinary neutrinos, may play a special role. They might even be the lightest /2,3/ new SUSY particles. This possibility arises naturally in models where the generation of mass terms for quark and lepton superfields is tied to their gauged quantum numbers like colour, electromagnetic charge or weak hypercharge $/ 4,5 /$.

In this note we study constraints on light scalar neutrinos from muon decay. Our analysis is based on the contribution to the decay amplitude shown in Fig.l, which has previously been considered by Kane and Rolnick in connection with unpolarized tau decay $/ 6 /$.

Using standard techniques / $7 /$ one obtains from the amplitude

$$
T=(\sqrt{2} g)^{2} \overline{U_{e}(p)} \frac{\not l-k_{1}}{\left(q-k_{1}\right)^{2}-M_{\tilde{W}}^{2}} \frac{1}{2}\left(1-\gamma_{5}\right) U_{\mu}(q)
$$

(where $q, p, k_{l}$ denote the momenta of the muon, electron, and $\tilde{\nu}_{\mu}$, respectively) the differential decay spectrum

$$
\begin{gathered}
\frac{d \Gamma \tilde{W}}{d x d(\cos \theta)}=\frac{G_{0}^{2} m_{\mu}^{5}}{3 \cdot 2^{7} \pi^{3}} \varepsilon x^{2}\left(1-\frac{r}{1-x}\right)^{3}[(3-x)-(1+x) \cos \theta] . \\
(1-\cos \phi) \theta(1-\Gamma-x)
\end{gathered}
$$

which has to be added to the standard differential decay rate due to $W$ exchange. The quantities $G_{0}, \varepsilon$, and $r$ are defined by

$$
G_{0}=\frac{\sqrt{2}}{8} \frac{g^{2}}{M_{W}^{2}}, \quad \varepsilon=\left(\frac{M_{W}}{M_{\tilde{W}}}\right)^{4}, \quad r=\left(\frac{m_{\tilde{v}_{\mu}}}{m_{\mu}}\right)^{2} .
$$

$x$ is the usual reduced energy variable 


$$
x=\frac{E_{e}}{E_{e}^{\max }} \simeq 2 \frac{p \cdot g}{m_{\mu}},
$$

$\theta$ is the emission angle of the electron relative to the muon spin, $\phi$ is the angle between the electron spin and its momentum, in the muon rest frame.

We restrict ourselves to the case $m_{e}, m_{v_{e}} \ll m_{\mu}$, assuming that a hierarchy of scalar neutrino masses would follow the pattern expected for ordinary neutrinos.

The energy spectrum which one obtains from (2) after integration over $\theta$, is in agreement with the result of ref./6/. Combining eq. (2) with the ordinary contribution involving an intermediate $W$-vector boson $/ 7 /$, we finally obtain

$$
\begin{aligned}
& \frac{d \Gamma}{d x d(\cos \theta)}=\frac{G_{0}^{2} m_{\mu}^{5}}{3 \cdot 2^{7} \pi^{3}} x^{2}\{(3-2 x)+(1-2 x) \cos \theta \\
& \left.+\varepsilon \theta(1-r-x)\left(1-\frac{r}{1-x}\right)^{3}[(3-x)-(1+x) \cos \theta]\right\} \quad(1-\cos \phi)
\end{aligned}
$$

Eq. (3) yields the total rate

$$
\Gamma=\frac{G_{0}^{2} m_{\mu}^{5}}{3 \cdot 2^{6} \pi^{3}}\left\{1+\varepsilon f_{0}(r)\right\},
$$

with

$$
f_{0}(r)=\frac{1}{2}(1-r)\left(3+47 r+1 l r^{2}-r^{3}\right)+6 r(2+3 r) \text { ln } r .
$$

We note first that the electron is fully polarized in a direction opposite to its momentum. Therefore the recent measurements of the electron polarization /8/ give no constraints on the new decay mode (2). As to the parameters $\rho, \delta, \xi$, we analyze eq. (3) by comparing it to the standard parametrization $/ 7 /$, (summing over the electron spin),

$$
\begin{aligned}
\frac{1}{\Gamma} \frac{d^{2} r}{d x d(\cos \theta)} & =x^{2}\left\{\left[6(1-x)+\frac{4}{3} \rho(4 x-3)\right]\right. \\
& \left.+\xi \cos \theta\left[2(x-1)+\frac{4}{3} \delta(3-4 x)\right]\right\} .
\end{aligned}
$$


2. In the case of vanishing $\tilde{\nu}_{\mu}$ mass, $r=0$, the comparison of eq. (3) to eq. (6) yields

$$
\rho=\frac{3}{4}\left(1+\frac{1}{2} \frac{\varepsilon}{1+\frac{3}{2} \varepsilon}\right) ; \delta=\frac{3}{4}\left(1-\frac{3}{2} \frac{\varepsilon}{1+\frac{3}{2} \varepsilon}\right) ; \quad \xi=1+2 \frac{\varepsilon}{1+\frac{3}{2} \varepsilon} .
$$

The parameters $\rho$ and $\delta$ being found very close to $\frac{3}{4}$ we see that $\varepsilon$ must be very small so that the denominators in eqs.(7) can be replaced by 1 . Solving formally for $\varepsilon$, the experimental values of $\rho$ and $\delta / 9 /$ would give $\varepsilon \simeq(4.5 \pm 6.9) \times 10^{-3}$ and $\varepsilon \simeq-(4.5 \pm 7.6) \times 10^{-3}$, respectively, yielding two Gaussians at equal and opposite positions with approximately the same standard derivation $\sigma$. The product of the two yields a Gaussian centered at zero and with $\sigma^{\prime}=\sigma / \sqrt{2}$. As $\varepsilon$ is positive, by definition, we normalize the branch of this latter Gaussian for positive argument to one and calculate from this the mean value of $\varepsilon$. We find $\langle\varepsilon\rangle=\sigma / \sqrt{\pi} \simeq 3.9 \times 10^{-3}$. Taking this as the upper limit for $\varepsilon$ we obtain a lower limit on the wino mass, viz.

$$
M_{\tilde{W}} / M_{W}>4 \text { (from } \rho \text { and } \delta \text { measurements combined). }
$$

We do not make use of the only existing measurement of $\xi, P_{\mu} \xi=0.975 \pm 0.015$, $/ 10 /$ because this result seems obscured by insufficient knowledge of the muon polarization $P_{\mu}$. In fact, as $\xi \approx 1+2 \varepsilon$ with positive $\varepsilon$, this parameter is the most sensitive to the wino contribution: Supposing we knew $\xi=1+0.001$ this would yield an improvement of the bound (8) to $M_{\tilde{W}} / M_{W}>7.1$. An experiment aiming at this level of accuracy is presently in preparation $/ 11 /$.

On the other hand, the combination $\left(\xi \frac{\delta}{\rho}\right)$ which was recently determined to very high accuracy $/ 12 /$ gives no useful constraint on $\varepsilon$ because, from eqs. (7), $\left[\left(\xi \frac{\delta}{p}\right)-1\right]$ is quadratic in $\varepsilon$.

3. For the case $r \neq 0$, we simulate the exact distribution (3) by fitting it to the expression (6) with effective parameters

$$
\rho_{\text {eff }}=\frac{3}{4}(1+\Delta \rho), \quad \delta_{\text {eff }}=\frac{3}{4}(1+\Delta \delta), \quad \xi_{\text {eff }}=1+\Delta \xi .
$$


The least squares fit can be evaluated analytically and leads to the following results

$$
\begin{aligned}
& \Delta \rho=\frac{\varepsilon}{l+\varepsilon f_{o}(r)} f_{\rho}(r), \\
& \Delta \delta=\frac{\varepsilon}{l+\varepsilon f_{o}(r)} f_{\delta}(r), \\
& \Delta \xi=\frac{\varepsilon}{l+\varepsilon f_{0}(r)} f_{\xi}(r),
\end{aligned}
$$

with $f_{0}(r)$ as given in eq. (5) above and with

$$
\begin{aligned}
& f_{\rho}(r)=(1-r)\left(\frac{1}{2}+\frac{77}{2} r+\frac{40}{7} r^{2}-\frac{4}{7} r^{3}\right)+\frac{3}{7} r\left(38+69 r-4 r^{2}\right) \ln r, \\
& f_{\delta}(r)=-\frac{3}{2}(1-r)(1+5 r)-3 r(2+r) \ln r, \\
& f_{\xi}(r)=(1-r)\left(2+37 r+\frac{35}{2} r^{2}+\frac{1}{2} r^{3}\right)+3 r\left(6+11 r+2 r^{2}\right) \ln r .
\end{aligned}
$$

The functions $f_{\rho}(r), f_{\delta}(r)$, and $f_{\xi}(r)$, are shown in figs.2,3 and 4, respectively, as a function of $m_{\tilde{v}_{\mu}} / m_{\mu}=\sqrt{r}$. It is easy to see that even with the additional degree of freedom $r, \varepsilon$ cannot be larger than, say a few $10^{-2}$. Therefore, we may again neglect the term $\varepsilon f_{0}(r)$ in the denominator of eqs.(9) as compared to 1 , so that $\Delta \rho, \Delta \delta, \Delta \xi$ are given by the universal functions (10)-(12), multiplied by $\varepsilon$.

Let us suppose $\rho$ were known to be $3 / 4$ within standard deviation $\sigma$. For a given $r$ we would then obtain an upper bound on the quantity $\varepsilon f_{\xi}(r)<\frac{\sigma \sqrt{2}}{\sqrt{\pi}}$, applying the analogous procedure as above. The lower limit on $M_{W} / M_{W}$ now depends on the mass ratio $m_{\tilde{\nu}_{\mu}} / m_{\mu}=\sqrt{r}$ as indicated in fig. 2 . Similarly, if $\delta$ were known to be $3 / 4$ within $\sigma=0.001$, or if $\xi$ were known to be 1 within the same $\sigma$, we would obtain the $r$-dependent bounds on $M_{W} / M_{W}$ as indicated in figs. 3 and 4 , respectively.

In general, we note that $\xi$ is the most sensitive parameter for small $r$, followed by $\delta$. At larger values of the $\tilde{\nu}_{\mu}$-mass, say $\sqrt{\Gamma}>0.35$, $\rho$ is slightly more sensitive than $\delta$, whilst $\xi$ quickly becomes insensitive to the wino diagram. In all cases the quantity $\left(\xi \frac{\delta}{\rho}\right)$ remains practically unaffected: 
For $r=0$ it is of order $\varepsilon^{2}$, for $r \neq 0$ it must be found to be $\sim 1$ if obtained from a measurement near $x \simeq 1$ because then the wino process does not contribute at $x=1$. Clearly, if improved data for more than one parameter become available, the combined analysis will yield even better bounds on $M_{W} / M_{W}$.

4. We have looked into the structure of the mass matrix which yields the wino masses via spontaneous symmetry breaking. In the simplest class of models $\varepsilon$ is always smaller than or equal to $l$. However, it is also possible to modify the mass matrix such as to obtain values for $\varepsilon$ larger than 1 . In the context of light scalar neutrinos the results from $\mu$-decay clearly exclude the latter possibility as well as the case where the wino and the $W$ are degenerate. For the case of vanishing s-neutrino masses present data lead to the conclusion $M_{\tilde{W}} \tilde{M_{W}}>4$. This limit is expected to be improved even further by the results of current precision experiments. For increasing mass of $\tilde{\nu}_{\mu}$ (but still below $m_{\mu}$ ), these bounds become somewhat weaker. However, unless $m_{\tilde{v}_{\mu}}$ is larger than $m_{\mu} / 2, M_{\tilde{W}}$ is still bound to be sizeably larger than $M_{W^{*}}$

A large mass splitting between the $W$ and its fermionic partner $\hat{W}_{\text {is }}$ unlikely to occur in models in which the Fermi scale of weak interactions is related to the supersymmetry breaking scale. The forthcoming new measurements of $\xi, \delta$ and $\rho$ with much increased accuracy will impose strong constraints on the possible existence of light scalar neutrinos and, in case a deviation from the canonical " $V$-A" values is established, will shed light on an important sector of supersymmetric physics. 


\section{References}

/1/ For recent reviews, see:

J. Ellis, in Proc. of the 1983 Int. Symp. on Lepton and Photon Interactions at High Energies (Cornell Univ., Ithaca, 1983);

5. Ferrara, in Proc. of Int. Europhysics Conf. on High Energy Physics (Rutherford Lab., U.K., 1983).

/2/ L.E. Ibáñez, Phys.Lett. 137B (1984)160.

13/ J.S. Hagelin, G.L. Kane and S. Raby, preprint LA-UR/83-3711.

14/ W. Buchmüller and D. Wyler, Phys.Lett. 121B(1983)32l.

/5/ W. Buchmüller, R.D. Peccei and T. Yanagida, Phys. Lett. 124B(1983)67

/6/ G.L. Kane and W.B. Rolnick, Nucl.Phys. B232(1984)21.

/7/ F. Scheck, "Leptons, Hadrons and Nuclei", North-Holland, Amsterdam (1983), sec.6.2

F. Scheck, Phys.Rep. 44(1978)187.

/8/ F. Corriveau et al., Phys.Rev. D24(1981)2004, Phys.Lett. 129B(1983)260.

/9/ Review of particle properties; Phys.Lett. 111B(1982)1

/10/ V.V. Akhmanov et al., Yad.Fiz. 6(1967)316 (engl. transl. Sov.J. of Nucl.Phys. 6 (1968) 230 .

/11/SIN-proposal R-80.09, W. Fetscher (spokesman).

/12/ J. Carr et al., Phys.Rev.Lett. 51(1983)627. 


\section{Figure Captions}

Fig. 1: Diagram describing the wino exchange contribution to $\mu^{-} \rightarrow \mathrm{e}^{-}$decay.

Fig. 2: The function $f_{\rho}(r)$, eq. (10), vs. $m_{\nu_{\mu}} / m_{\mu} \equiv \sqrt{\Gamma}$, that describes the deviation of the effective Michel parameter $\rho_{\text {eff }}$ from 3/4. The numbers and open points indicate bounds on the mass ratio $M_{W} / M_{W}$ of wino to $W$ mass which would be obtained if $\rho$ were measured to be 0.75 within one per mil.

Fig. 3: The function $f_{\delta}(r)$, eq. (11), vs. $m_{\tilde{v}}^{\tilde{v}} / m_{\mu} \equiv \sqrt{\Gamma}$, describing the deviation of $\delta_{\text {eff }}$ from 3/4. The numbers and open points indicate bounds on $M_{W} \sim M_{W}$ which would be obtained if $\delta=0.75 \pm 0.1 \%$

Fig. 4: The function $f_{\xi}(r)$, eq. (12), vs. $m_{v_{\mu}} / m_{\mu} \equiv \sqrt{r}$, describing the deviation of the effective asymmetry parameter $\xi_{\text {eff }}$ from 1 . The numbers and open circles indicate bounds on $M_{W} / M_{W}$ which would be obtained if $\xi=1 \pm 0.1 \%$. 


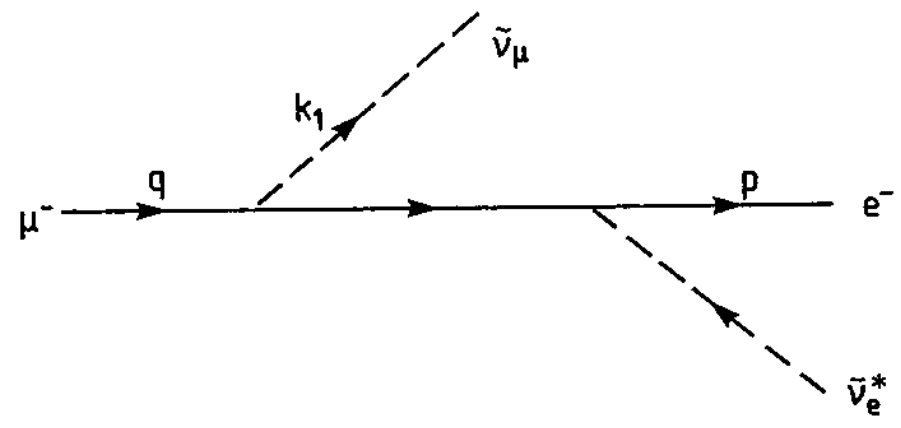

- Figure 1 -

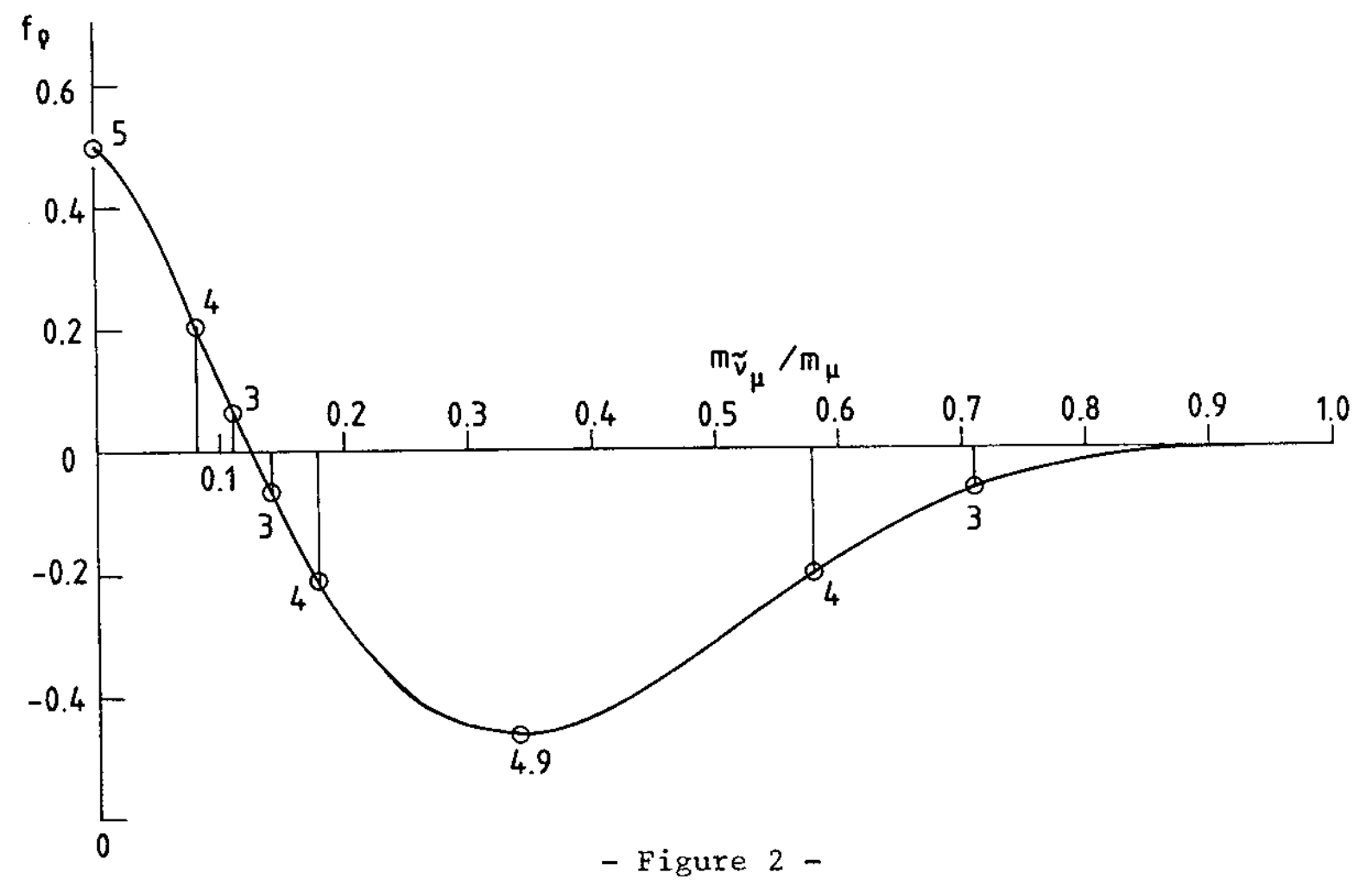




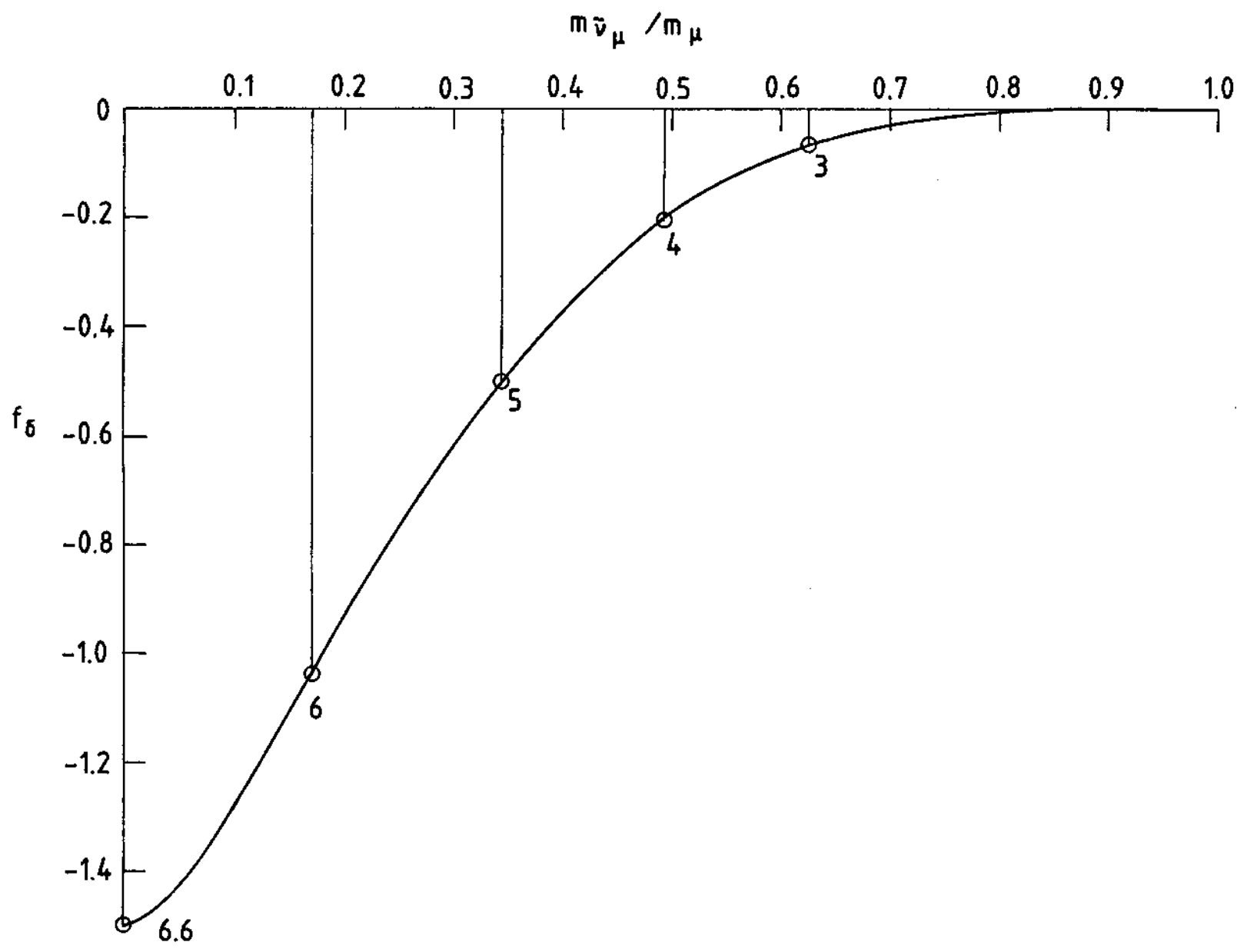

- Figure 3 - 


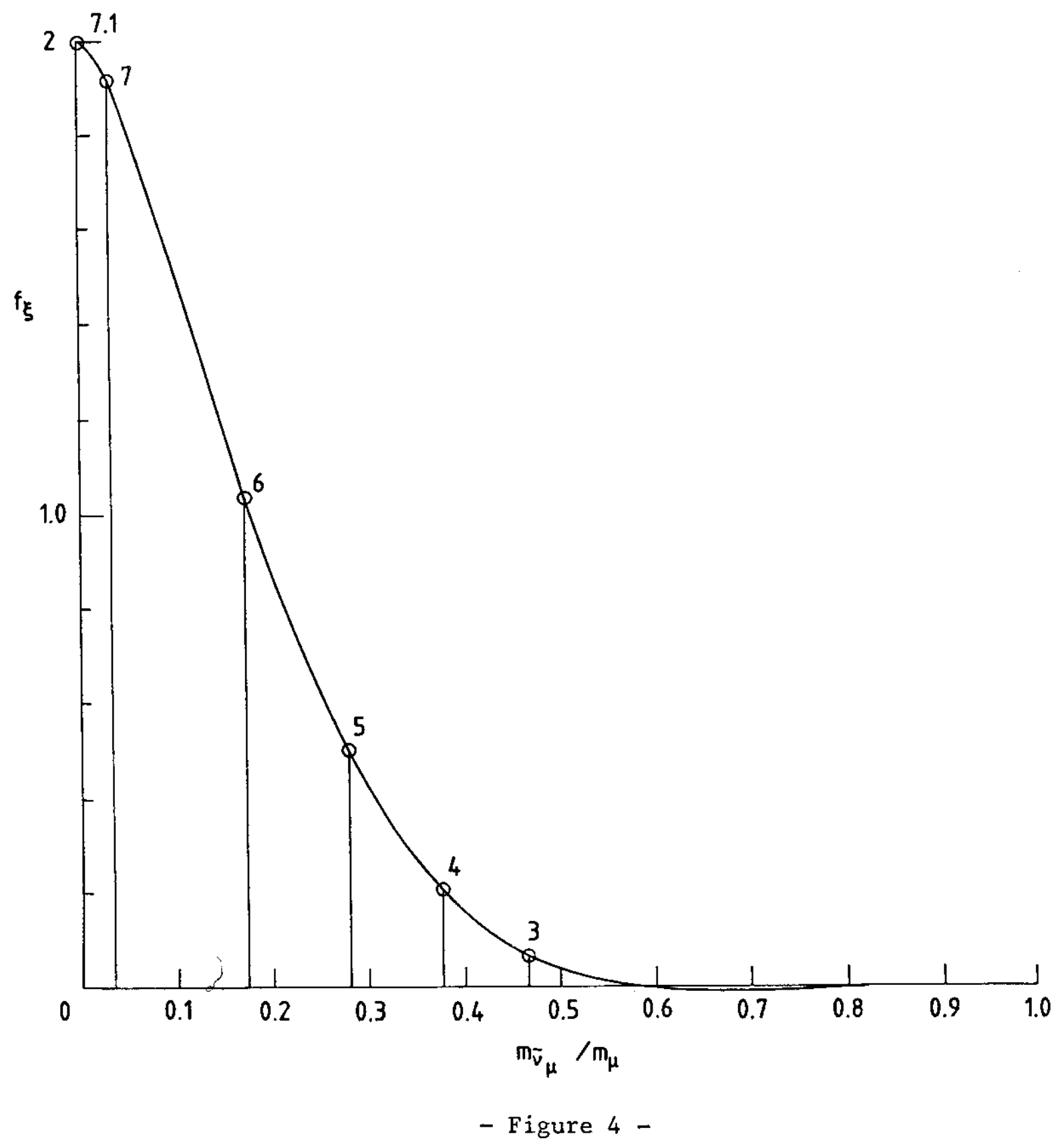

